\title{
LOGGING AND ANALYZING LONG-TERM MOBILE USER BEHAVIOR
}

\author{
Hui-Yin Wu \\ National Chengchi University \\ NO.64, Sec.2, ZhiNan Rd., Wenshan District, Taipei City 11605, \\ Taiwan (R.O.C) \\ 100462010@nccu.edu.tw \\ Wen-Hung Liao \\ National Chengchi University \\ NO.64, Sec.2, ZhiNan Rd., Wenshan District, Taipei City 11605, \\ Taiwan (R.O.C) \\ whliao@cs.nccu.edu.tw \\ Po-Ming Chen \\ National Chengchi University \\ NO.64, Sec.2, ZhiNan Rd., Wenshan District, Taipei City 11605, \\ Taiwan (R.O.C) \\ radination@gmail.com \\ Chih-Yu Hsu \\ National Chengchi University \\ N NO.64, Sec.2, ZhiNan Rd., Wenshan District, Taipei City 11605 , \\ Taiwan (R.O.C) \\ easyflys@gmail.com \\ Tsai-Yen Li \\ National Chengchi University \\ NO.64, Sec.2, ZhiNan Rd., Wenshan District, Taipei City 11605, \\ Taiwan (R.O.C) \\ li@nccu.edu.tw
}

\begin{abstract}
With the rapid evolution of mobile devices, the usage patterns of applications on these devices also change over time. Recently, the topic of how user behavior and usage patterns of applications has adapted to new mobile devices such as smartphones has attracted much attention. This work presents an experimental platform that can $\log$ and analyze all user
\end{abstract}


operations on a smartphone used in a real-life context over long periods of time. The collected data are transmitted to a server periodically whenever an Internet connection is available. Analytic tools such as charting, log query, and real-time log pattern analysis have also been developed to facilitate the study of user behavior. This paper illustrates the functionalities of the proposed platform through several examples and a sample case study.

Keywords: Mobile Devices, User Log Analysis, Mobile User Behavior

\section{INTRODUCTION}

The rapid development of mobile devices and communication infrastructure encourages the development of various smartphone applications. The enhanced functionality through these applications has transformed the smartphone into a mini computer, providing wireless services such as web access, location-based service (LBS), and social networking. Novel services are being developed to take advantage of the evolving mobile platform. Thus, how people have adopted the smartphone and developed their own usage patterns deserves further investigation.

There are many approaches to studies of user behavior. Typical studies on user experiences are often designed and carried out in a controlled experimental environment where users are instructed to perform a certain task while researchers examine their behavior. An experimental session is usually videotaped, and corresponding key events during the process are marked for further identification of the motive behind a specific user operation. A survey questionnaire is often used to collect background information on the participants as well as subjective ratings of the system under experiment. The disadvantage of this kind of experimental design is that the tasks to be performed by the users need to be clearly identified in advance, and they are usually limited to those that can be accomplished within a limited amount of time. The design of the experimental environment is also crucial to avoid unnecessary discomfort or pressure on the participants. In addition, traditional approaches have limitations for the study of long-term daily usage patterns. It is therefore highly desirable to develop new methodologies to understand user behavior and assess user preference over a longer period of time. Existing practices can be complemented by an experimental platform that provides convenient logging services and appropriate analytical tools for studying these logs on mobile devices. 
Smartphone use can be rather complex in everyday life. A single application on a smartphone may require several functional modules to work together seamlessly. For example, a photo-taking activity may consist of retrieving location information from the GPS system, taking the picture, and uploading the picture to a social network site. To give another example, placing a call may involve entering the phone number manually, selecting it from his/her contact list, or finding recent contacts from the call history. Although these actions all aim to place a phone call, action patterns may carry different meanings under varying usage contexts. Studies of these behaviors are crucial for improving smartphone design and applications; however, they cannot be easily conducted in a controlled laboratory. In addition, many mobile phones feature built-in social network services. Investigation of the dynamics and characteristics of mobile social networks also calls for the development of new and powerful log collection and analysis tools.

Smartphones are becoming dominant because of their openness and extensibility. Among the most popular mobile operating systems, Android OS features the greatest extensibility and can be further customized for different phone brands and models. Its flexibility in modifying and supplementing extra functions for the system allows one to implement a monitoring application to keep track of user operations. In our previous work $^{1}$, we took advantage of this openness to develop a log collection service that executes in the background and regularly records and uploads detailed user operations to a database. The user logs are transferred to a remote server when an Internet connection is available. In this paper, we extend the work reported in Chen et al. ${ }^{2}$ and present the tools that we have designed to facilitate the collection and analysis of the log data. We use several examples and a comprehensive case study to demonstrate the potential uses of these tools for analyzing mobile user behavior.

The rest of this paper is organized as follows. In Section 2, we briefly review related work pertaining to long-term log collection and analysis. Section 3 presents the log collection and analysis services implemented in this research. Section 4 describes three demonstrative examples and a comprehensive application of using these proposed services. Section 5 concludes with a look at future research directions.

\section{RELATED WORK}

In the past few years, we have witnessed the gradual increase of research in the field of mobile user behavior. In Eagle and Pentland ${ }^{3}$, the authors analyze long-term location-based information collected through 
Bluetooth devices to gain deeper understanding of user personal behavior. Specific details of the mobile operations carried out by users can be retrieved through a background user-log collection program, as demonstrated in Kärkkäinen, Vaittinen, and Väänänen-Vainio-Mattila ${ }^{4}$. Individual usage patterns are recorded in the form of log data, which encompass daily activities such as text messaging, phone calls, photo-taking, game play and other leisure activities. We use a similar log-collecting approach in this paper but with a broader goal to provide a platform that integrates hardware resources, software design and user group management. In this way, researchers from different academic backgrounds can cooperate to explore new topics in interdisciplinary areas. In Pramudiono et al. ${ }^{5}$, we note an example of search engine and location-aware service analysis on mobile devices by analyzing the access logs of a tool called Mobile Info Search (MIS), which provides location aware search facilities while obtaining user query data for further analysis. As proposed in Baeza-Yates ${ }^{6}$, web logs are recorded for user behavior analysis and to provide suggestions for improving the query effectiveness of the search engine. We use a similar log collection concept to obtain user behavior logs. Nonetheless, we seek to record all user behavior on mobile devices and emphasize the benefits of such a study for future interface and software design.

The online game community is another place where large amounts of log data are collected, mainly for recording different sessions throughout the gameplay. In Ducheneaut et al. $^{7}$, the authors create a database to store character profile information such as character ID and location. The information is collected through a client-side user interface. Analysis of the relationship between characters and groups (or guilds) reveals the evolution of online communities from a social network perspective. The authors designed a tool called the Social Dashboard to monitor group dynamics in real-time. This concept is incorporated our research to develop a similar web-based log monitoring tool that allows researchers to efficiently access the overall status of the collected data. This ensures smoother operations and convenience throughout the progress of the experiment.

The data obtained through the log collecting service are useful for qualitative analysis on user behavior, as proposed in Kort and $\mathrm{Poot}^{8}$. In Hilbert and Redmiles ${ }^{9}$, the authors provide a framework for automated usage analysis of user interfaces to include functionalities in synch and search, transforming event streams, analysis, visualization, and evaluation support. On our platform, we implement several key components of this framework on the mobile device logging platform including log charting services for graph visualization and statistic analysis, log query service for searching and statistic evaluation, and log real-time monitor for user 
behavior pattern interpretations. This can provide a basis for both quantitative and qualitative research as proposed in Leontjev ${ }^{10}$.

\section{THE EXPERIMENTAL PLATFORM}

In this section, we describe the structure of our experimental platform, which consists of the log collection service that was implemented in our previous work ${ }^{1}$ and the analysis tools that have been developed more recently. We first describe the initial setup of our experiment, namely, the recruiting of volunteers. We then describe the details of the log collection service, including the data flow, log table format and service policy. Finally, we introduce the three services that we currently provide on this platform for $\log$ analysis.

Our experimental platform consists of a collection of smartphones with the Android operation system, a log collection program that is pre-installed on the phones, and a community of users who borrow our smartphones or use their own phones to participate in the long-term experiment in a living lab. The participants were recruited on a quarterly basis. There are 30 participants in the experiment reported in this work. Most participants took part for the whole period, which lasted one year. They were not given any constraints on phone usage except for installing the logging program at the very beginning of the experiment. Therefore, all data collected in the experiment reflect their usage of the mobile phone in daily life.

Because of the open nature of Android operating system, we could utilize the functions provided by the Location Manager to record position information from localization services such as GPS, Wi-Fi, and 3G. As for system logs, there is no corresponding API to directly access the low-level statistics. Instead, we make use of the logcat tool included in the Android SDK to obtain raw data and then parse and filter the desired portion according to our needs.

All log data generated as a result of user operations are transferred and stored in a central repository so that researchers can retrieve certain sections of the data according to their specific interests. For example, researchers who would like to analyze the correlation between the photo-taking behavior and the location where the photo was taken could gather all activities related to the camera application and the corresponding location information gathered through GPS. Researchers who are interested in investigating the dynamics of social behaviors need to define a set of terms containing all social networking activities of interest in order to retrieve data accordingly. The log database is updated incessantly and incrementally, and the proposed platform provides a convenient web-based user interface for 
registered researchers to quickly browse and query the log content, as further elucidated in the following section.

The log collection platform has three services in order to provide researchers with a simple and convenient interface for retrieving and analyzing the log data stored in our database: the log charting service, log query service, and the real-time monitor. The log charting service gives an overview of the data collected, which is useful in identifying possible trends and research questions. The log query and real-time monitor further provide tools to make specific queries for log files that match a certain term or pattern. The services are discussed in depth in the next section.

\section{ILLUSTRATION EXAMPLES}

Our main objective is to build an experiment platform which integrates hardware devices, software customization and user group management for researchers from different areas of expertise to conduct long-term observation as well as to collect and analyze mobile user behavior such as gaming, social interactions, web browsing, photo-sharing, etc. In the following subsections, we use three examples to illustrate how the services provided by the platform can be used to interpret the collected data and further analyze user behavior.

\subsection{Log Charting Service}

The Log Charting Service provides the researcher with a quick way to obtain an overview of the data collected from the users who have installed the Log Collector. This data includes application information, time stamp, usage frequency, etc. By providing this service, researchers can efficiently compare the user behavior of various applications, evaluate which applications are most popular among the group, and gain deeper insight into more recently released apps. Example activity and application charts generated by our tools appear in Figure 1. 


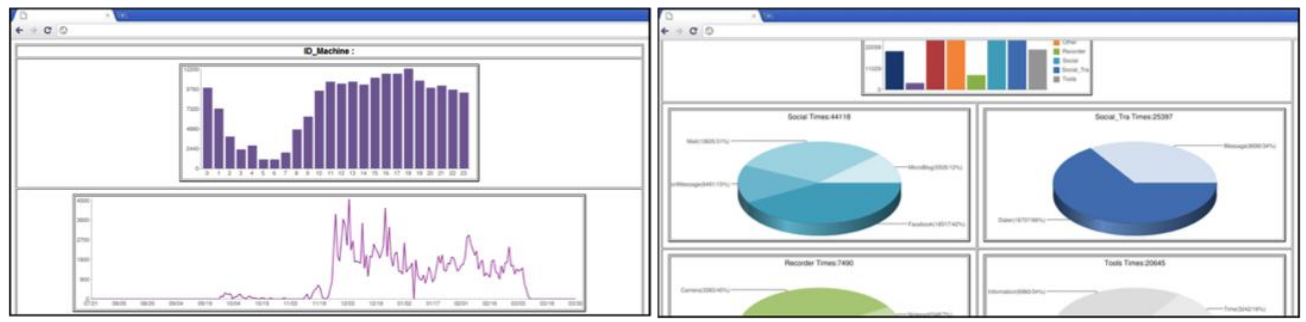

Figure 1. Left: User activity chart (top: over a 24-hour period; bottom: throughout the whole experiment period); Right: Charting the types of applications run by a specific user

Figure 2 is an example output result provided by the Charting Service. The example observes the time periods in which our participants use applications most frequently. By analyzing the figures produced by the Charting Service, a quick summary of the log files can be gained. The period of user log files obtained for analysis is from May 2011 to November 2011. The top figures show the number of activities at different times of day, and the bottom figure uses the size of a circle to indicate the number of activities for different times of each day. The researchers can easily grasp the general activity pattern for each day using this type of graph plot.

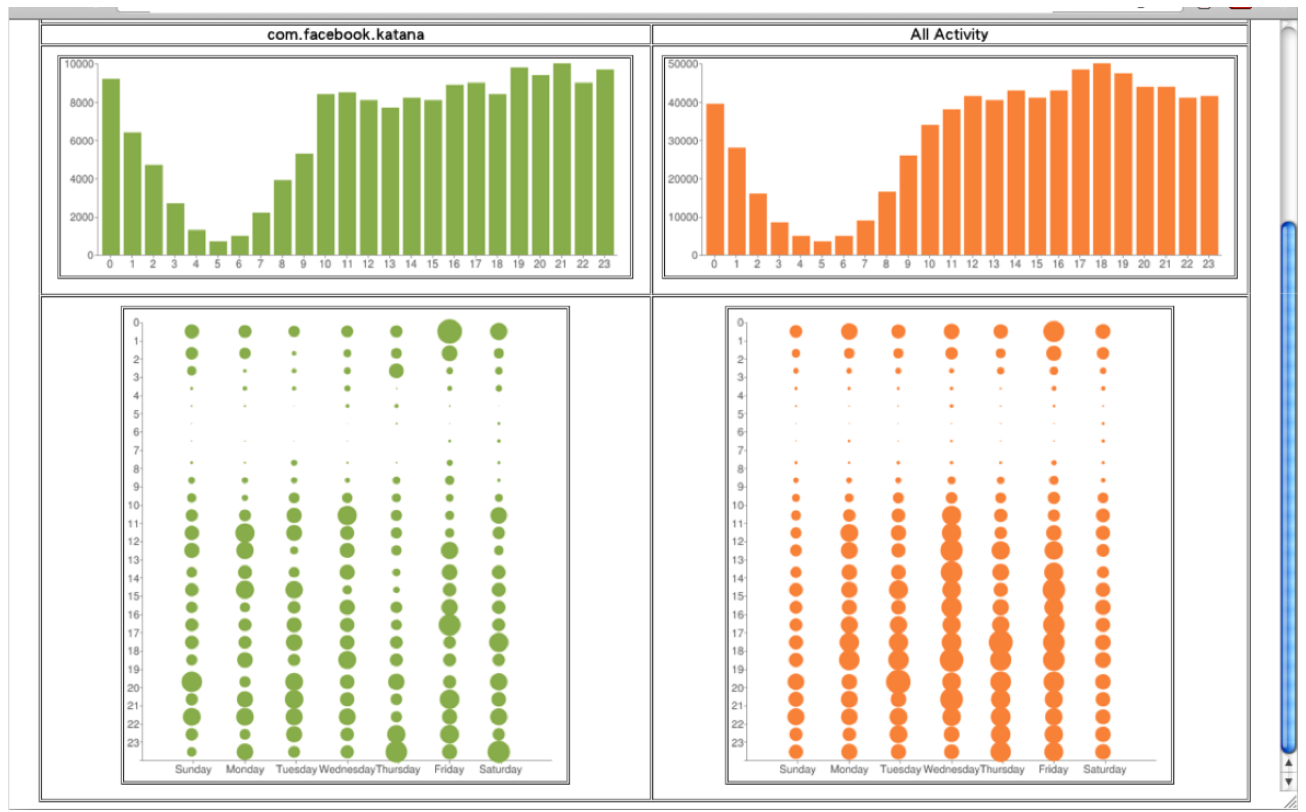

Figure 2. Top: The number of times that an app runs at each time of day; Bottom: The number of times that an app is run on each weekday, at different times of the day, and the period of usage 
In Figure 3, we show the usage of various applications over a six-month period. We can see that HTC launcher has a strong correlation with the applications that are executed, because the launcher app is used each time an application is closed and the mobile device returns to the home screen. Also, from the overall app data, we can note that Facebook is the most frequently used app, which confirms the importance of social network services for current smartphone users. Another very popular application that was recently released is the Line App. By using the Charting Service, the researcher can visualize the usage information and understand the life-cycle of an app for all users.

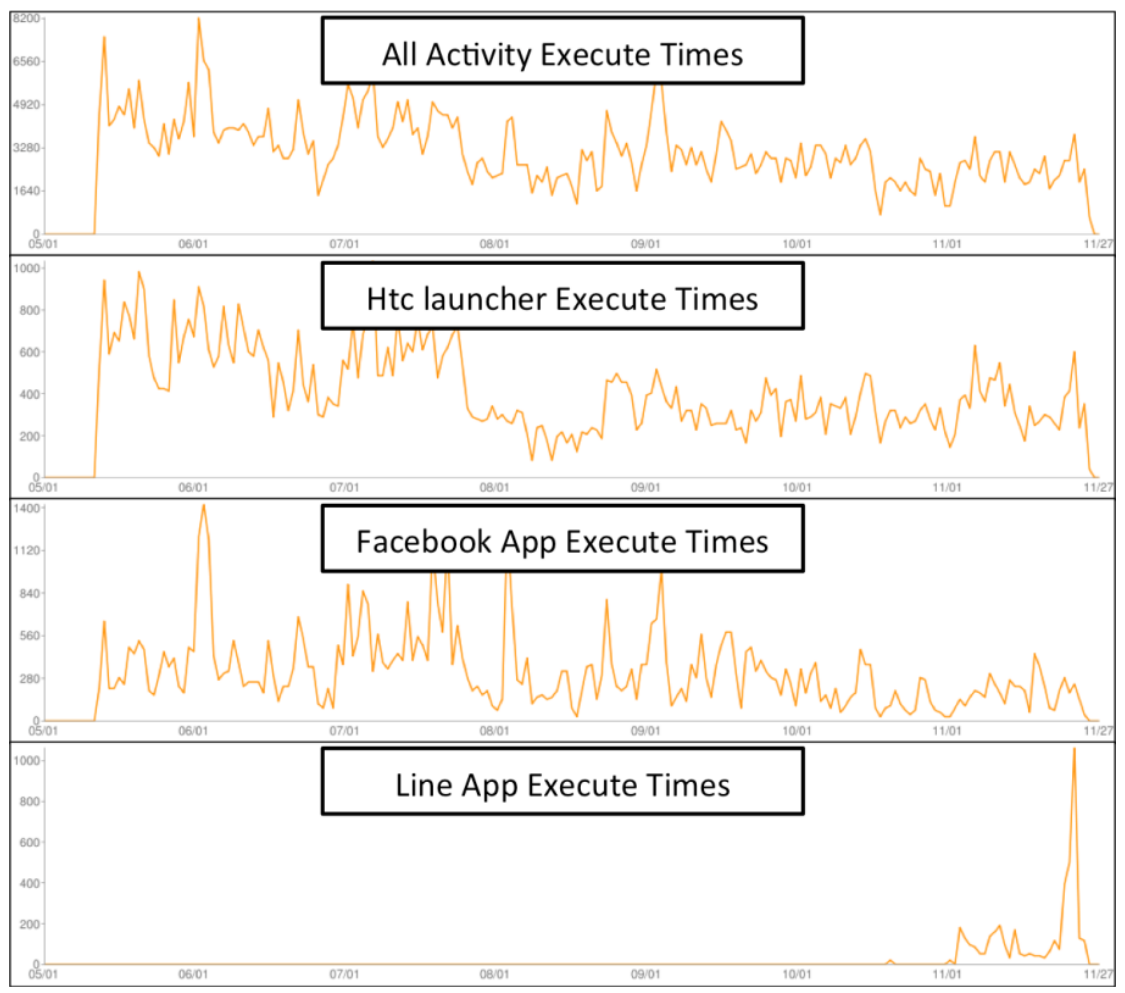

Figure 3. The number of times that each application is run throughout the course of a day

Although the above figures show the daily usage of different applications among users, they do not differentiate between workdays and weekends or holidays. With further analysis, deeper insight into the frequency of applications run at various points of time throughout the day can also be gained.

Figure 4 depicts the different usage of applications at various times of the day during each day of the week. Applications like the Dialer App are 
usually used on weekdays from 5PM to 6PM, when class ends. Compared to other days of the week, the Dialer App has most usage on Friday afternoons after class. Therefore, we can see that the Clock App and the Dialer App vary in usage at different times of the day, the former usually in the morning and late night and the latter in the afternoon. We can also observe that both of these apps are used more frequently on weekdays compared to weekends. It would be interesting to determine whether any other applications are more frequently used during weekends or holidays.

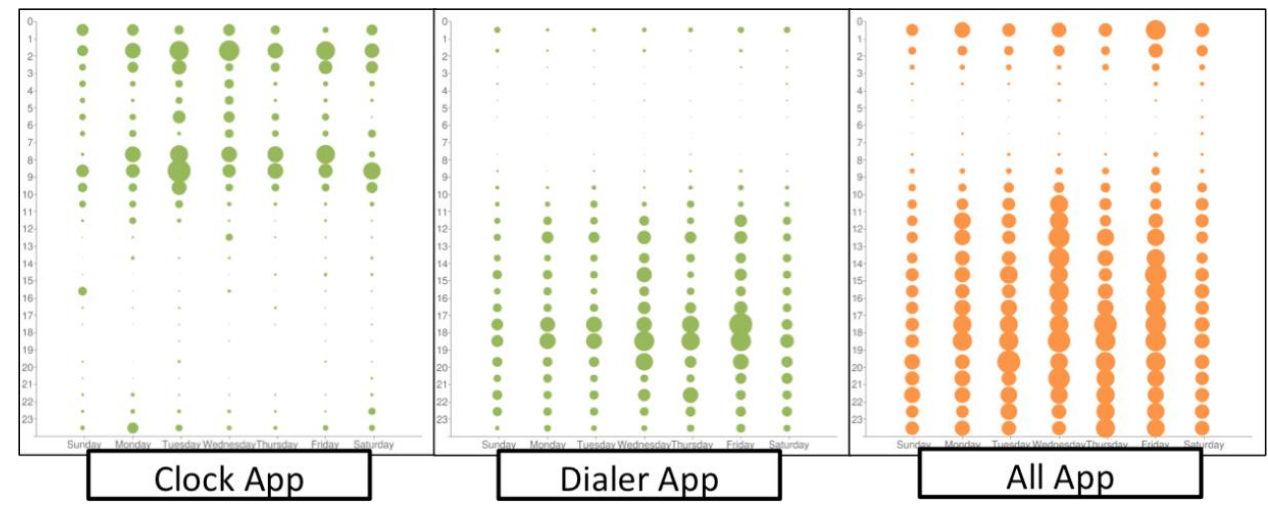

Figure 4. The usage distribution for all users based on day of the week, hour of the day and time that an application is run

Researchers can quickly compare the usage behavior between different categories of apps through the Charting Service. This provides a convenient platform to efficiently set search conditions and select applications that fit the topic of interest. However, there are still limitations to the platform in that the accuracy of its grouping of related applications is defined by the developer or Android market categories. Therefore, a robust solution to compare the usage of related applications is yet to be developed. Comparing user behaviors across similar applications at various times would be an interesting topic for future research. Generally speaking, we believe that this charting tool can serve as a starting point for discovering interesting research issues for further qualitative studies.

\subsection{Log Query Service}

It is also interesting to determine how users evaluate an application. Where did they gain access to it? How frequently is the application used? When does it come into need? How long is its span of interest? Does the user decide to uninstall it after it falls out of favor? Using the log files collected on the platform combined with the Log Query Service, some insights can be gained into the life cycle of applications. 
In this study, we examine the user log files of three communications related apps collected over a twelve-month period. The first application is a free text messaging app, while the second and third are free Internet phone applications, the former a long existing service and the latter relatively new. We compare the number of log files collected throughout a twelve-month period and examine the changes in the amount of usage.

Table 1. Usage comparison for the three apps

\begin{tabular}{ccc}
\hline & $\begin{array}{c}\text { Average logs } \\
\text { (Per month) }\end{array}$ & $\begin{array}{c}\text { Average Life Cycle } \\
\text { (Months) }\end{array}$ \\
\hline Text App & 476.41 & $8+$ \\
Voice App 1 & 31.28 & 1.67 \\
Voice App 2 & 142.09 & 2.22 \\
\hline
\end{tabular}

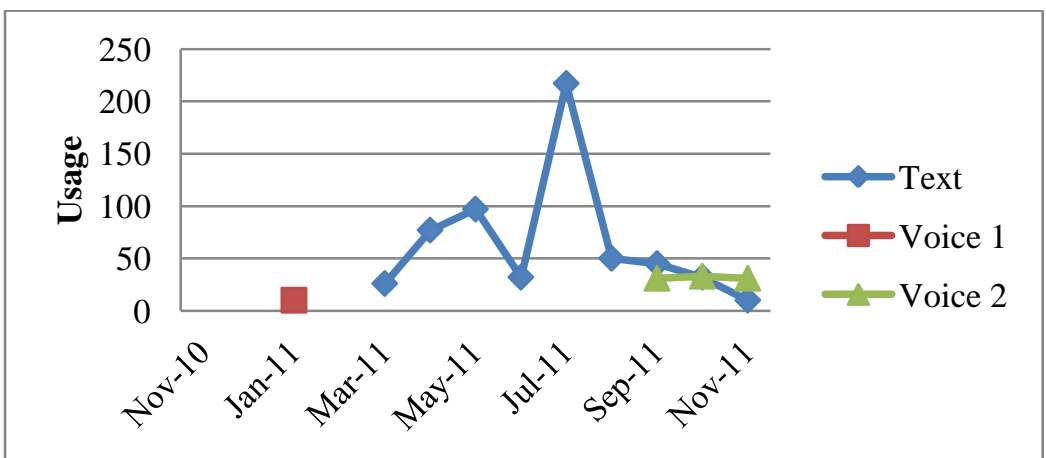

(a)

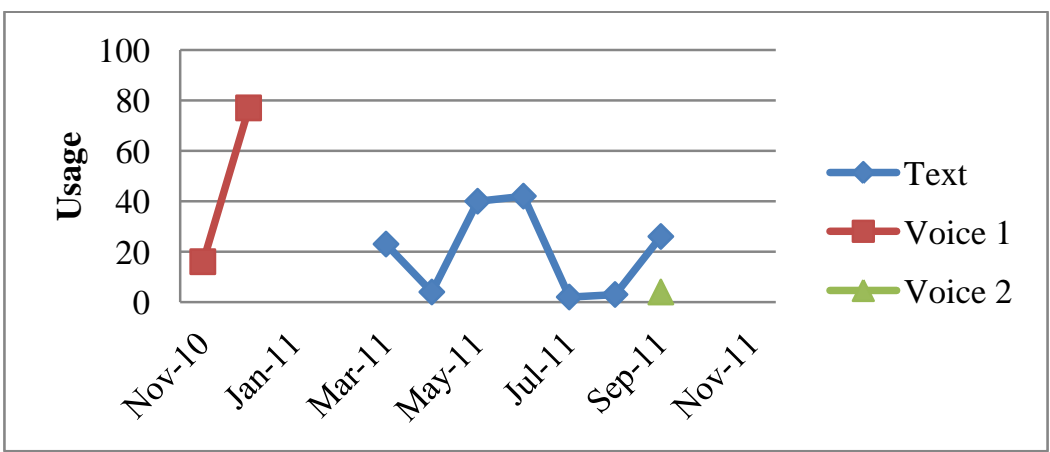

(b)

Figure 5. Comparison of $\log$ amounts for (a) User 1 and (b) User 2

A few observations are made from the collected data. First, we find that users prefer the text messaging app to voice messaging apps. Although use of the text messaging app is occasionally high and low, the app remains in 
longer usage from installation until the end of the experiment period, the shortest being three months and the longest over twelve months, with an average usage period of eight months. In comparison, the usage graph for the two Internet phone apps both have disconnected and scattered points of usage that do not last more than two months. Specifically, the first voice app has an average life cycle of 1.67 months spanning from just a few hours to three months, and the second has an average life cycle of 2.22 months. The comparison of the usage data is summarized in Table 1.

Because the time when the user downloads each app varies, it is difficult to define an absolute starting point that can apply to every user. However, if we compare the app usage of individual users, we can still observe similarities in the sequence of downloading and the period of usage (Figure 5).

Observing use of the text app, we can see that although the shape of the usage curves do not completely overlap, all curves exhibit similar trends for the four sample users (Figure 6).

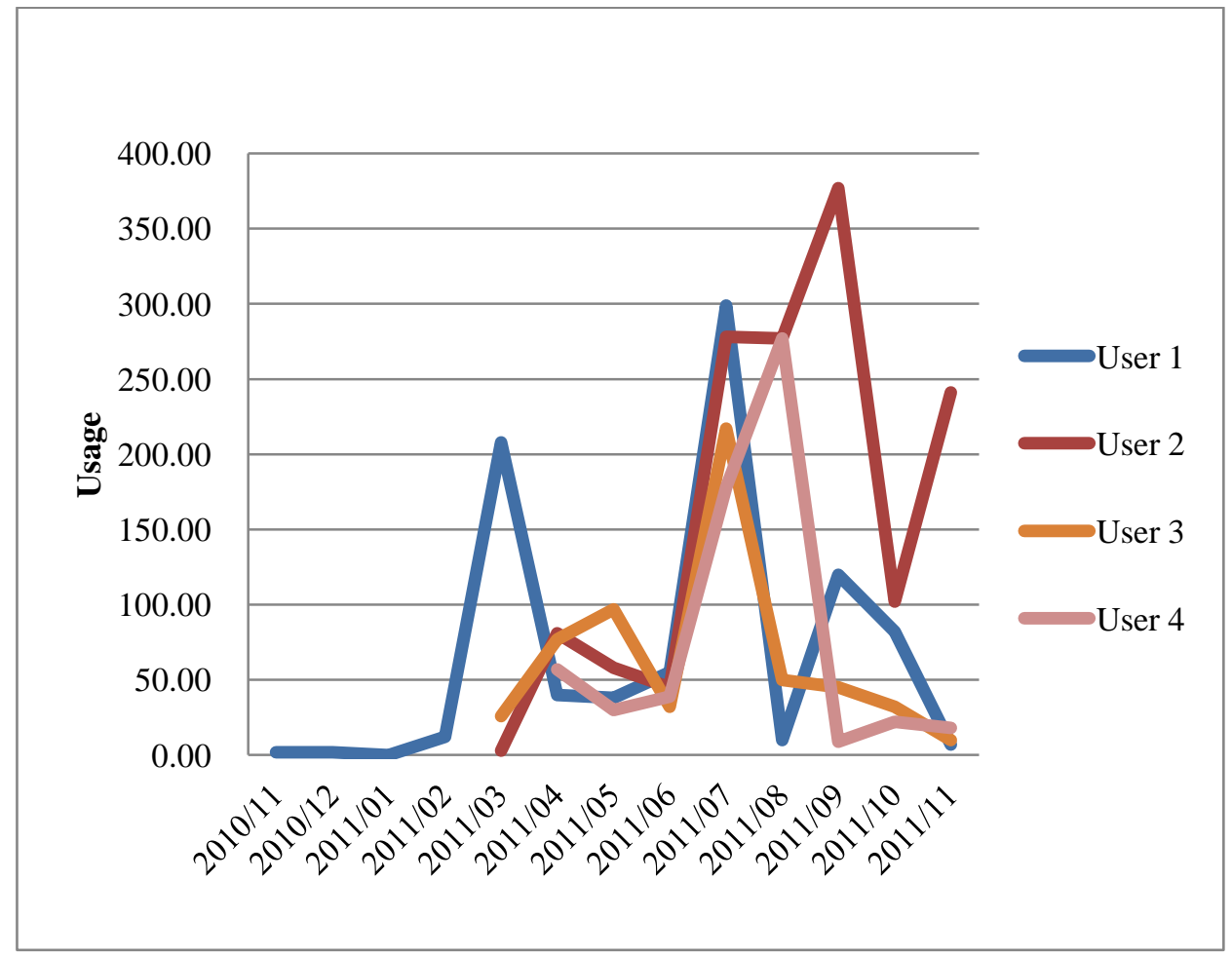

Figure 6. Usage information for four users of the text app 
In a more in-depth survey, User 3 (Figure 1) stated that there were three stages to the life cycle of app usage: initial experience, gradual balanced usage, and, if applicable, uninstalling unused applications. Using the text application as an example, in the initial stage, User 3 felt that the app was interesting, and this fascination led to more frequent usage. However, User 3 did not have texting habits prior to installing the app, so gradually the usage decreased after the freshness wore off. The irregularly high usage pattern in mid-summer occurred while on travel, when the need to contact family members and friends in a more efficient and inexpensive way arose. When asked about use of the other two apps after installation, User 3 stated that they were used infrequently, because few of his contacts used them; thus, there was no need to set them up. Since the apps were out of use for some period, and User 3 realized that memory space was limited, the unused apps were deleted.

In this case, we can observe that although all three apps are free services for communication purposes, their usage models vary greatly. The text app seems to be designed for more sustained use, while the two voice apps have a much shorter span of interest among users.

\subsection{Real-time Log Monitor}

In the literature, three different levels of user behavior analysis have been identified: activity, action, and operation ${ }^{10}$. An activity usually consists of a sequence of actions and is more related to motivation. An action is a logical unit with a clear goal, while an operation is the most fundamental physical unit. From the researcher's perspective, higher levels of description such as activity or action are of interest. However, a log item can only show us the operation level of the action performed. A sequence of logs constitutes higher-level actions and activities. Therefore, we need to establish the mapping between an event and its corresponding log sequence. If researchers wish to examine certain user behavior on smartphones by predicting the log pattern produced through a certain activity, they can immediately find the Log List of certain log patterns by manipulating the elements on the GUI with the help of the Log Real-time Monitor. In this fashion, it is much easier to define user behavior patterns and gain insight into the usage pattern.

In Figure 7, the $\log$ files related to dialing activities are marked. Through the Log Monitor service, the researcher can observe that com.android.phone .InCallScreen is the Activity users evoke when entering a phone or dial application. However, there are many possibilities for how the user evokes the call activity. For example, the user may manually dial the phone number through the number pad or via speed dial. Both methods 
achieve the same purpose of placing a call. Other applications may have similar scenarios. For example, for a photo-sharing activity, a single log file is insufficient to determine the whole course of activity. Therefore, a sequence of log files must be defined in order to produce a pattern that can be used as a query to obtain data for further analysis. For example, if a user chooses to place a call by searching through his/her contact list, the .InCallScreen operation is preceded by the com.android.htccontacts .ContactDetailLayerCarouselActivity operation. The call placing action is then initiated by two log activities instead of one. By using these two activities, we can define a user behavior pattern for placing a call.

\begin{tabular}{|c|c|c|}
\hline PKG_NAME & STATE & DATA \\
\hline \multicolumn{3}{|c|}{ com.androld.pnone Incaliscreen } \\
\hline com.android.vending & AssetBrowserActivity & NODATA \\
\hline com.google android talk & SigninglnActivity & NODATA \\
\hline $\begin{array}{l}\text { om.androld.phone } \\
\text { om android.htccontacts }\end{array}$ & $\begin{array}{l}\text { Incallscreen } \\
\text { ViewCallHistory }\end{array}$ & $\begin{array}{l}\text { tel.XXX-XXX-XXXX } \\
\text { NODATA }\end{array}$ \\
\hline com.htc.launcher & Launcher & NODATA \\
\hline com.htc.launcher & Launcher & NODATA \\
\hline com.htc.launcher & Launcher & NODATA \\
\hline com.htc.launcher & Launcher & NODATA \\
\hline $\begin{array}{l}\text { fom.androld.phone } \\
\text { om android.htccontacts }\end{array}$ & $\begin{array}{l}\text { Incaliscreen } \\
\text { ContactDetailLaverCarouselActivity }\end{array}$ & $\begin{array}{l}\text { content } / / / \text { com.androld.contacts/data/1888 } \\
\text { content///com.android.contacts/contacts/lookup/3989i60c75d0a0c9b3766/41. }\end{array}$ \\
\hline \multicolumn{3}{|c|}{ com.gau go.launcherex com.jiubang.ggheart.apps.desks.diy.GoLauncherNODATA } \\
\hline com.android.mms & ui.ComposeMessageActivity & content://mms-sms-v2/conversations/72?thread_id=true \\
\hline com android.htccontacts & BrowseLayerCarouselActivity & NODATA \\
\hline com.dropbox.android & activity.DropboxBrowser & NODATA \\
\hline com android. htccontacts & BrowseLayerCarouselActivity & NODATA \\
\hline $\begin{array}{l}\text { om.android.phone } \\
\text { om. android. } \text { htcdialer }\end{array}$ & $\begin{array}{l}\text { InCallscreen } \\
\text { Dialer }\end{array}$ & $\begin{array}{l}\text { tel:XXX-XXX-xxxX } \\
\text { NODATA }\end{array}$ \\
\hline com.whatsapp & Conversations & NODATA \\
\hline com. whatsapp & Main & NODATA \\
\hline
\end{tabular}

Figure 7. Log real-time monitor

Here is an explanation of deducing a user behavior pattern through the assistance of the Log Real-time Monitor to create a Log List. We analyze the log data produced based on six user scenarios.

Scenario 1 for Manually Inputting a Phone Number (Figure 8): When the expected receiver of the call is not in the contact list, or when the caller remembers the phone number, he/she may choose to use this method to place a call. 
- Manually Inputting the Phone Number
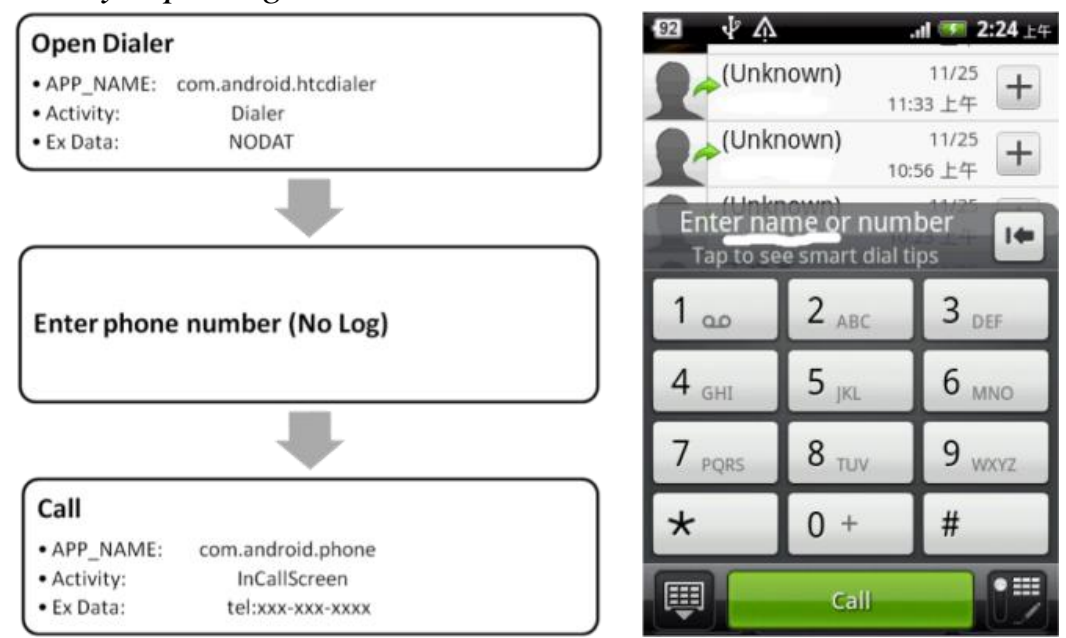

Figure 8. Left: Steps for placing a call by manually inputting a phone number; Right: Screen for manually inputting a phone number

Scenario 2 for Contact List (Figure 9): When the contacts and their phone numbers are saved prior to the call, the user is not familiar with the receiver's number, or when it has been some time since the number was dialed, the user may choose to search for the number through their contact list.

- Contact List

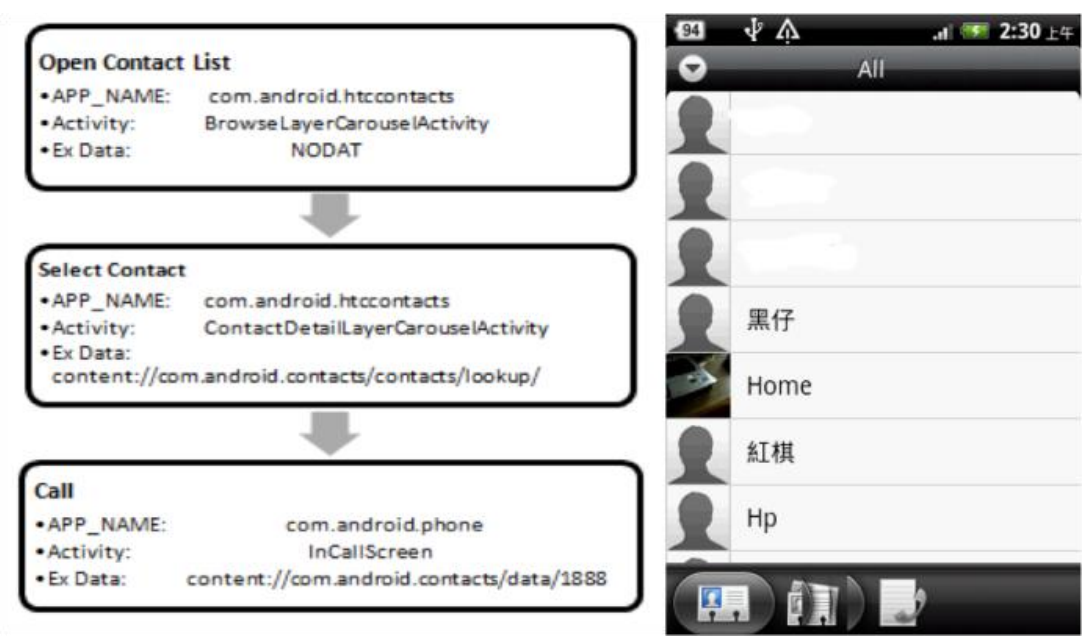

Figure 9. Left: Steps for placing a call through the contact list; Right: Screen for contact list 
- Call History
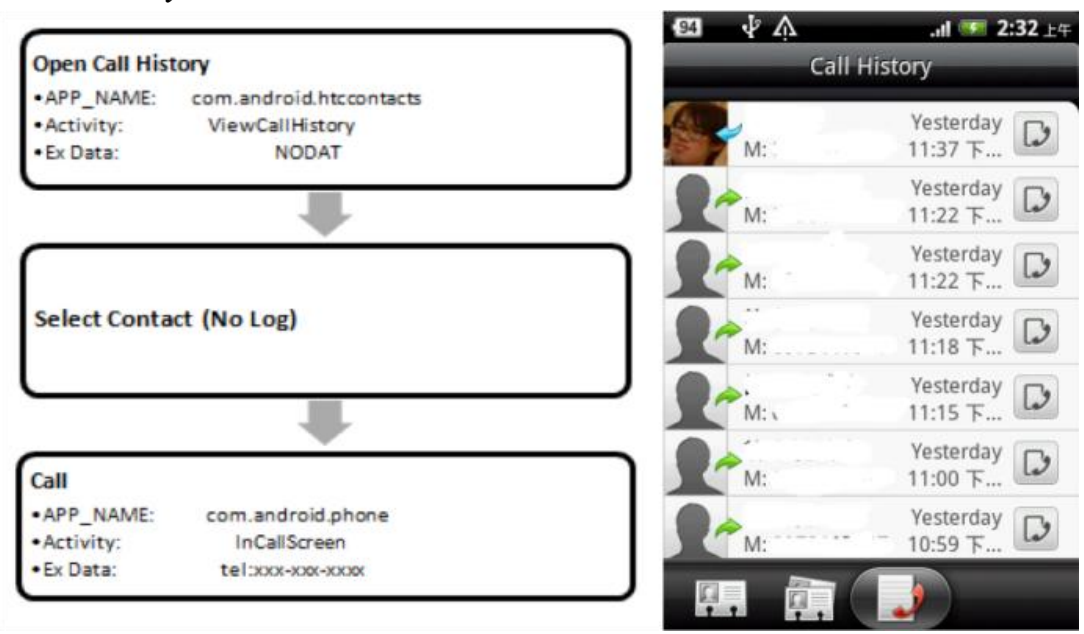

Figure 10. Left: Steps for placing a call through call history; Right: Screen for call history

Scenario 3 for Call History (Figure 10): When a contact has been called recently, the user can directly place a call by searching through the call history.

- Frequent Contacts

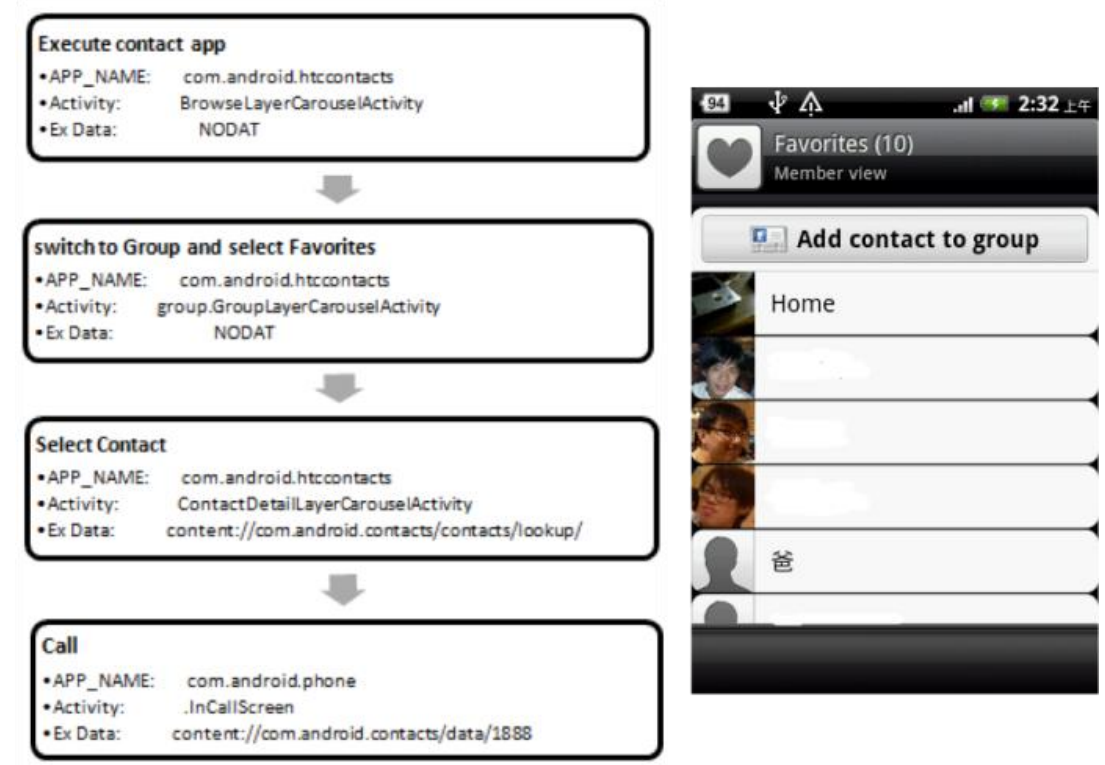

Figure 11. Left: Steps for placing a call through Favorites; Right: Screen for Favorites 
Scenario 4 for Frequent Contacts (Figure 11): When the receiver of the call has already been added to the Favorites list, the user can open the Favorites page to view a certain group of frequently-used contacts, which narrows down the search overhead.

Scenario 5 for Widget (Figure 12): When the user puts frequently used contacts in the widget management, they can dial through a home screen shortcut instead of opening their contact list, which differs from the use of Favorite lists in speed and convenience. This greatly reduces the select elapsed time before placing a call.

Scenario 6 for Call from Voice: The contact must already be in the contact list. When users cannot use usual methods to place a call (e.g. while driving), they may choose to use Android's voice recognition function instead. However, Android currently does not provide a good Chinese voice recognition system, so the log files for this method cannot yet be found.

\section{- Widget:}
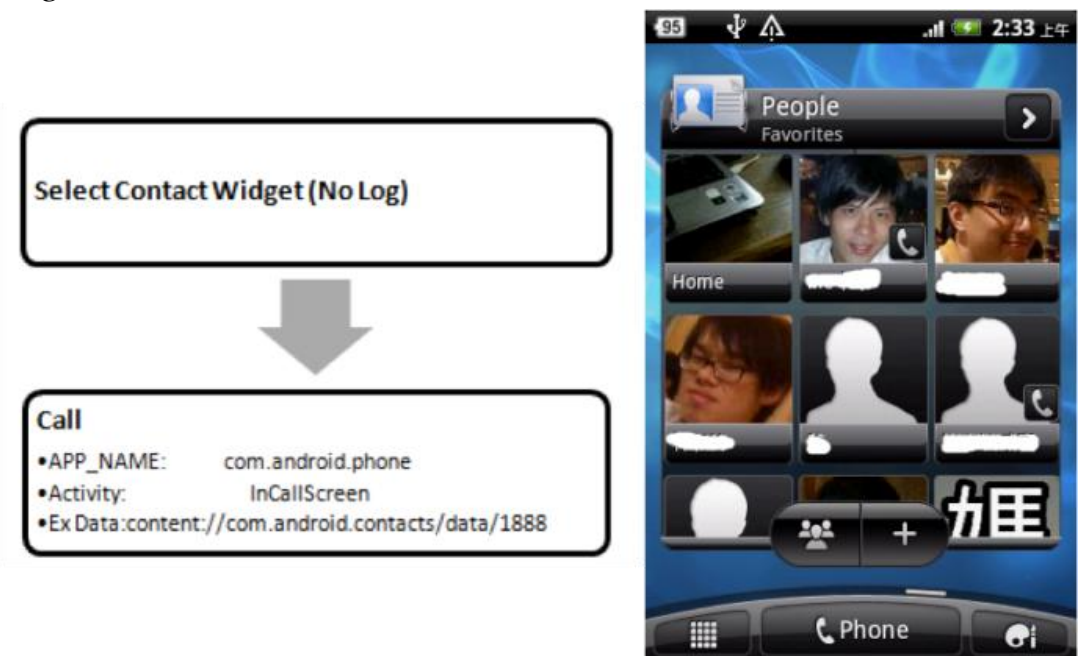

Figure 12. Left: Steps for placing a call through Widget; Right: Screen for Widget

By using the Log Real-time Monitor, we can define five log patterns for user behaviors. However, note that as indicated in Table 2, the log pattern for the widget scenario includes only a single log record. This kind of log pattern may cause difficulty in analysis. Moreover, although it takes four steps to place a call through Favorites, it is often still faster than searching through the Contact List. This may be because the user can find their desired contact much faster through the shorter Favorites List. However, we cannot calculate the time that it takes for the user to search 
through their contact list since no logs are produced for the searching process. Therefore, the activity log records cannot completely determine the length of time to carry out an activity.

By combining user behavior studies with the Log Real-time Monitor, researchers can quickly and efficiently find related log lists and define user operations to generate a $\log$ pattern. This saves the time and trouble of searching through a huge amount of $\log$ data for relevant information. However, although the Log Monitor provides a convenient interface for researchers to search and understand user behavior patterns and also greatly increases the efficiency, it is still limited compared to the variety of applications that can be found on the Android Market. Apart from the default applications on the phone, other well-designed applications with similar functionalities can prospectively replace the function of the default application. This may cause much difficulty when analyzing User Log records. Moreover, if the user has a certain unique habit of usage that cannot be predicted prior to analyzing, it may cause misinterpretation of the data. A critical issue for future research would be on how the Log Real-time Monitor can reduce such errors through more accurate definition of user patterns.

Table 2. Steps for placing a call and the relative number of applications, activities, and logs evoked

\begin{tabular}{lcccc}
\hline & Step & $\begin{array}{c}\text { Used } \\
\text { App }\end{array}$ & $\begin{array}{c}\text { Used } \\
\text { Activity }\end{array}$ & Log \\
\hline Manual enter phone number & 3 & 2 & 2 & 2 \\
Contact List & 3 & 2 & 3 & 3 \\
Favorite contact & $\mathbf{4}$ & $\mathbf{2}$ & $\mathbf{4}$ & $\mathbf{4}$ \\
Call history & 3 & 2 & 2 & 2 \\
Widget: & $\mathbf{2}$ & $\mathbf{1}$ & $\mathbf{1}$ & $\mathbf{1}$ \\
\hline
\end{tabular}

\subsection{Comprehensive Application}

In this section, we illustrate some functionalities of our service platform with a sample case study in which the researcher is interested in knowing if a user is accustomed to sharing photos on Facebook immediately after taking pictures. Traditional survey-based or interview-oriented approaches may have limitations due to the lack of concrete data to support quantitative analysis. Such experiments cannot be conducted in a lab setting due to the need for long-term observation. Using the proposed service 
platform, researchers can search for activities related to 'camera' and 'Facebook' in a specified time interval and establish the causality to examine the usage pattern. To begin, the researcher defines photo-sharing activity as a sequence of user actions. This sequence is repeated several times, and the corresponding log list is observed using the Real-Time Log Monitor. The researcher looks for consistency and possible noise in the generated $\log$ to properly model the activity. When the experiment commences, participants are told about the period of this experiment and are encouraged to operate their mobile phones using default settings (for example, using the built-in camera application to take pictures or using Facebook, but not other social network platforms, to share photos.) During the experiment, researchers can check for general user activities using the charting function. They can also retrieve partial data to perform preliminary analysis. The results shown in Figure 13 were obtained with a user group of size 25 over a 3-month period. Here, instant sharing is acknowledged if the user uploads the picture to Facebook within 10 minutes of shooting.

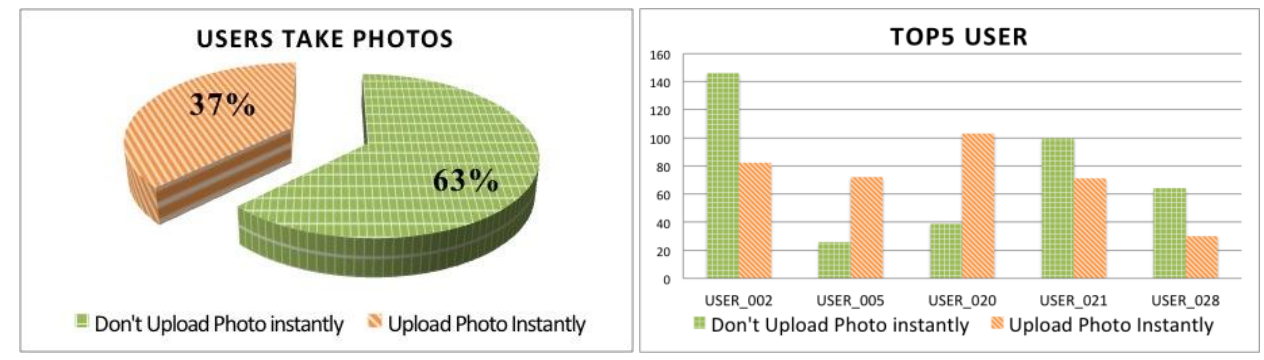

Figure 13. Statistics of photo-sharing behavior

According to the statistics shown, 37\% of the photos taken by the participants are shared on Facebook without much delay. If we turn our attention to the five most active photographers, we observe two distinct types of users: \{USER_005, USER_020\} are more social than \{USER_002, USER_021, USER_028\}. Of course, there exists other ways of categorizing the users if we incorporate more information from the log data.

This case study demonstrates how the three services can be used to carry out a simple user behavior survey. The Log Query and Real-Time Log Monitor provide a simple way to access and obtain data related to the research question, namely, the photo-taking action of the camera app and the uploading action of the Facebook app. The log charting service is then used to provide a quick overview of the data. 


\section{CONCLUSIONS AND FUTURE WORK}

In this paper, we have presented a service platform for logging and analyzing user behaviors on mobile devices. The objective of this research is to provide a new direction for conducting research on user behaviors by taking advantage of the openness of the Android operating system to gather objective statistics based on user operations on mobile devices. We have also developed tools to facilitate the analysis of such user behaviors. Three examples are shown to illustrate the use of these tools to analyze and compare user behaviors and discover prospective research issues for further studies. We hope that the development of this experimental platform will enable the advent of novel research methods for analyzing user behavior on mobile devices.

We are in the process of deriving automated methods to discover usage patterns from the log data. Since similar activities may result in different operation patterns under different application contexts, we hope to develop a new description language to describe the usage pattern in order to facilitate automatic extraction of these patterns from the log. We would also like to conduct further analysis on the current collected user log to discover more interesting research issues for further qualitative studies and benefit the design of mobile applications.

\section{ACKNOWLEDGEMENT}

This work was supported in part by the Top University Project (2010-2012) of the Ministry of Education granted to National Chengchi University.

\section{REFERENCES}

[1] P-.M. Chen, C-.H. Chen, W-.H. Liao, and T-.Y. Li, A service platform for logging and analyzing mobile user behaviors. Edutainment Technologies. Educational Games and Virtual Reality/Augmented Reality Applications Lecture Notes in Computer Science, 6872, p78-85, 2011. http://dx.doi.org/10.1007/978-3-642-23456-9_15.

[2] P-.M. Chen, H-.Y. Wu, C-.Y. Hsu, W-.H. Liao, and T-.Y. Li, Logging and analyzing mobile user behaviors. Paper Presented at the International Symposium on Cyber Behavior, Taipei, Taiwan, February 10-12, 2012.

[3] N. Eagle, and A. Pentland, Reality mining: Sensing complex social systems. Personal Ubiquitous Computing, 10(4), p255-268, 2006. http://dx.doi.org/10.1007/s00779-005-0046-3. 
[4] T. Kärkkäinen, T. Vaittinen, and K. Väänänen-Vainio-Mattila, I don't mind being logged, but want to remain in control: A field study of mobile activity and context logging. In G. Fitzpatrick, S. Hudson, K. Edwards, and T. Rodden (Eds.), Proceedings of the 28th international conference on Human factors in computing systems (p163-172). New York, NY, USA: ACM Press, 2010. http://dx.doi.org/10.1145/1753326.1753351.

[5] I. Pramudiono, T. Shintani, K. Takahashi, and M. Kitsuregawa: User behavior analysis of location aware search engine. In A. Denise Williams (Ed.), Proceedings of the 3rd International Conference on Mobile Data Management (p139-145). Singapore: IEEE Press, 2002. http://dx.doi.org/10.1109/MDM.2002.994398.

[6] R.A. Baeza-Yates. Applications of web query mining. Advances in Information Retrieval Lecture Notes in Computer Science, 3408, p7-22, 2005. http://dx.doi.org/10.1007/978-3-540-31865-1_2.

[7] N. Ducheneaut, N. Yee, E. Nickell, and R.J. Moore, The life and death of online gaming communities: A look at guilds in world of warcraft. In D. Gilmore (Ed.), Proceedings of the SIGCHI conference on Human factors in computing systems (p839-848). New York, NY, USA: ACM Press, 2007. http://dx.doi.org/10.1145/1240624.1240750.

[8] J. Kort, and H. de Poot, Usage Analysis: Combining logging and qualitative methods. In C. Gale (Ed.), Proceedings of the 2005 CHI Conference on Human Factors in Computing Systems (Extended Abstracts) (p2121-2122). Vienna: ACM Press, 2005. http://dx.doi.org/10.1145/1056808.1057117.

[9] D.M. Hilbert, and D.F. Redmiles, Extracting usability information from user interface events. ACM Computing Surveys 2000, 32(4), p384-421, 2000. http://dx.doi.org/10.1145/371578.371593.

[10] A.N. Leontjev, Activity, consciousness, and personality. Prentice Hall: Englewood Cliffs, 1978. 